\title{
Spatial and Temporal Wave Functions of Photon
}

\author{
D.L. Khokhlov \\ Sumy State University \\ R.-Korsakov St. 2, 40007 Sumy, Ukraine \\ Tel: 380-542-332-558Ｅ-mail: dlkhokhl@rambler.ru
}

\begin{abstract}
The quantum mechanical model of electromagnetic waves is considered. The photon as a particle moves with the speed of light with respect to the CMB frame. The spatial wave function of the photon is introduced as a wave accompanying the photon, with an infinite speed of spreading throughout the whole space at some moment of time. The temporal wave function of the photon emitted (absorbed) by the electron is defined through the temporal wave function of the electron (virtual photon associated with the electron) at some point of space. The Michelson-Morley experiment is studied with use of both the spatial and temporal wave functions of photon, and the null result being obtained in both the cases.
\end{abstract}

Keywords: Wave function of photon, Michelson-Morley experiment

\section{Introduction}

Special relativity (Pauli, 1958) restricts the speed of propagation of the particles to the speed of light $c$. On the other hand, quantum mechanics (Dirac, 1958) admits of the spatial wave function defined instantaneously in the whole space. Here comes the conflict between special relativity and quantum mechanics. The free will theorem was proven (Conway \& Kochen, 2006) from which it follows that any theory admitting wave function collapse cannot be made relativistically invariant. The problems of special relativity and quantum mechanics are discussed elsewhere (Nikolic, 2007). The phenomenon of entanglement gives an example of the wave function defined instantaneously between two space-like separated states. Quantum correlations between two space-like separated states detected in Bell experiments suggest a violation of relativistic locality, e.g. (Shimony, 2004) and references therein. Bell experiments with the moving detectors are discussed in (Suarez, 2003) and references therein. Nonetheless, all events are supposed to lie within the past light cone in accordance with the special relativity causality. An example is the theory of direct action at a distance (Wheeler \& Feinmann, 1949). In addition, the theory of two steps light is worth to be mentioned (Whitney, 2007).

Within the quantum mechanics framework electromagnetic waves have dual particle-wave behaviour. The Copenhagen interpretation of quantum mechanics regards the particle-wave duality through the complementarity principle and the Heisenberg uncertainty principle, e.g. (Busch \& Shilladay, 2006) and references therein. In the de Broglie-Bohm interpretation both the wave function and the particle position are fundamental entities (Bohm \& Hiley, 1993; Holland, 1994). We shall not follow any interpretation literally but rather quantum mechanics in common sense.

In (Khokhlov, 2008) the quantum mechanical model of electromagnetic waves is considered in which the photon as a particle propagates with the speed of light while the spatial wave function of the photon spreads out with an infinite speed. The Michelson-Morley experiment was studied within the framework of this model. The conclusion is that the phase difference between the source and receiver defined through the spatial wave function does not depend on the velocity of the frame that may explain the null result of the Michelson-Morley experiment. In the present paper we shall introduce the temporal wave function of photon and study the Michelson-Morley experiment with use of the temporal wave function of photon. To this end, a quantum mechanical model of light to handle non-locality is considered in (Cardone \& Mignani, 2004).

The Maxwell-Lorentz equations describe classical electromagnetic wave propagating with the velocity $c$. It is reasonable to think that electromagnetic wave propagates with the velocity $c$ with respect to a privileged frame. If some frame moves with the velocity $v$ with respect to a privileged frame then one can expect that electromagnetic wave propagates with the velocity $\vec{c}-\vec{v}$ with respect to the moving frame. The Michelson-Morley experiment was suggested to determine the velocity of electromagnetic wave with respect to a moving frame, with the earth being taken as a moving frame. However, the Michelson-Morley experiment 
(Pauli, 1958) yielded the null result. Special relativity (Pauli, 1958) explains the null result of the Michelson-Morley experiment with the Lorentz transformation for coordinates of space and time.

For completeness, the result of the Michelson-Morley experiment was non-null but rather small to explain the velocity of the earth. The Michelson-Morley experiment was repeated by several authors, and the modern scheme of the experiment utilizes optical resonators to stabilize the laser frequency. Discussion of the results of the Michelson-Morley experiments may be found in (Munera, 1998; Cahill \& Kitto, 2003; Consoli \& Costanzo, 2004; Gezari, 2009). Special relativity (Lorentz invariance) successfully passed many tests (Zhang, 1997). Nonetheless, the search for violation of Lorentz invariance in precision experiments and astrophysical observations is under way to constrain the modern physical theories, e.g. (Pospelov \& Romalis, 2004) and references therein.

Quantum mechanics requires a privileged reference frame (ether) and absolute time to prevent backward causality (Bell, 1989). The cosmic microwave background radiation (CMB) is usually taken as a privileged reference frame (Lineweaver et al, 1996). The speed of quantum information in a privileged reference frame with use of entangled photons was measured (Salart et al, 2008), and the experimental bound is more than $10^{7} c$ in the Geneva reference frame and more than $10^{4} c$ in the CMB frame. Experiments with entangled relativistic massive particles are in agreement with the non-relativistic quantum mechanics that admits a privileged reference frame, see discussion in (Rembieliski \& Smolinski, 2009) and references therein. The Lorentz approach introduces a privileged reference frame in the special relativity. The common belief is that the Einstein and Lorentz approaches are mere two interpretations of the same theory (Duffy, 2004). The special relativity with a privileged frame and absolute time is considered in (Selleri, 1996; Levy, 2003; Hatch, 2004; de Abreu \& Guerra, 2008). In addition, Galilean invariant ether theory based on Hertz's progressive wave equations is considered in (Thornhill, 2004; Dunning-Davies, 2005; Christov, 2006), Galilean invariant local ether theory in (Su. 2001), the theory of magnetic light propagation in (Spavieri, 2006). Also, it was shown (Kholmetskii, 2003) that the Faraday induction law is Lorentz non-invariant.

\section{Spatial wave function of photon}

Consider electromagnetic wave with the vector potential $\vec{A}$ in the Euclidean space and absolute time of a privileged reference frame. We shall choose the cosmic microwave background radiation (CMB) as a privileged reference frame. The Maxwell-Lorentz equations for the electromagnetic wave yield a solution as a plane monochromatic wave (Landau \& Lifshitz, 1976)

$$
\vec{A}=\vec{A}_{0} e^{-i \phi}
$$

with the phase

$$
\phi=\omega t-k r
$$

where $\omega$ is the frequency, $k$ is the wave vector.

In quantum mechanics (Dirac, 1958) one can consider electromagnetic wave as a bunch of photons, with the momentum and energy given by respectively

$$
p=\hbar k \quad E=\hbar \omega
$$

where $\hbar$ is the Planck constant. One can conceive the photon as a particle exhibiting wave behaviour. In quantum mechanics the wave eq. (1) is thought of as a wave function of photon associated with a single photon. For photons the Heisenberg uncertainty principle holds true

$$
\Delta p \Delta r \geq \hbar / 2 \quad \Delta E \Delta t \geq \hbar / 2 .
$$

In view of eq. (3), the Heisenberg uncertainty relations restrict the wave function of photon in space and time.

Consider the photon as a particle with the momentum $p$ at the time $t$ (Khokhlov, 2008). Introduce the wave function of the photon with the wave vector $k=p / \hbar$. In the stationary state the momentum of photon is fixed. Then, the uncertainty in momentum is $\Delta p=0$, the uncertainty in wave vector is $\Delta k=\Delta p / \hbar=0$. From the Heisenberg uncertainty relations eq. (4), it follows that the uncertainty in the space coordinate is $\Delta r=\infty$. This means that one cannot specify the space coordinate hence one can consider the wave function of the photon with the wave vector $k$ in the whole space at the time $t$. The spatial wave function of photon spreads out instantaneously over the whole space with an infinite speed $v_{\infty}=\infty$. Hence, the uncertainty in time is $\Delta t=\Delta r / v_{\infty}=0$. This means that the spatial wave function of photon cannot be specified in time. The spatial wave function of photon is not suitable to define the speed of light. Instead, one can consider propagation of the photon as a massless particle with the speed $c$ with respect to the CMB frame. 
Consider propagation of a photon between the source and receiver (Khokhlov, 2008). We shall regard the case when the region of propagation of the photon is much more than the wave length of the photon $\Delta r>>\lambda=1 / k$. Then, one can think of the photon as a point like quasi classical particle propagating with the speed $c$ with respect to the $\mathrm{CMB}$ frame. In view of the above reasoning one can define the spatial wave function of the photon in the whole space at the time of reception. Then, the phase difference between the source and receiver is a distance between the source and receiver at the time of reception

$$
\Delta \phi=\phi_{r}\left(t_{r}\right)-\phi_{s}\left(t_{r}\right)=k\left[r_{r}\left(t_{r}\right)-r_{s}\left(t_{r}\right)\right] .
$$

Unlike the phase of the classical wave defined through the space coordinate of the source at the time of emission $r_{s}\left(t_{e}\right)$ the phase of the spatial wave function of photon is defined through the space coordinate of the source at the time of reception $r_{s}\left(t_{r}\right)$. When determining the distance between the source and receiver at one and the same moment of time it does not depend on the velocity of the frame. Hence, the phase difference between the source and receiver eq. (5) does not depend on the velocity of the frame.

Consider the Michelson-Morley experiment in a frame moving with the velocity $v$ with respect to the CMB frame. Suppose that electromagnetic field (photon) moves with the velocity $c$ with respect to the CMB frame, independently of the source (receiver). Then, the travel time is a function of the velocity of the frame, with the maximum difference of travel time between two legs for two-way travel being $\Delta t=(l / c)\left(v^{2} / c^{2}\right)$ where $l$ is the length of the leg. According to quantum mechanics (Dirac, 1958) a single photon interferes with itself. The wave function of the photon is a superposition of the waves specified along two different legs, with the photon as a particle moving along one of the legs. In view of eq. (5), the phase difference between the source and receiver is a distance between the source and receiver at the time of reception. If the lengths of the legs are not the same there is a phase shift between two waves specified along two different legs $\Delta \phi=k\left(l_{2}-l_{1}\right)$. When determining the distance between the source and receiver at one and the same moment of time it does not depend on the velocity of the frame. Hence, the phase difference between the source and receiver does not depend on the velocity of the frame. Thus, there is no phase shift due to the velocity of the frame between two waves specified along two different legs that can explain the null result of the Michelson-Morley experiment without invoking the Lorentz transformation. To this end, the same idea was stated elsewhere (Sato, 2004).

\section{Temporal wave function of photon}

Consider the electron as a particle in the point of space $r$. One can think of the energy of the electron in terms of excitations being virtual photons. We shall define the stationary state of the electron through the stationary state of the virtual photon with the energy $E$. Introduce the temporal wave function of the electron through the temporal wave function of the virtual photon with the frequency $\omega=E / \hbar$. In the stationary state the energy is fixed then the uncertainty in energy is $\Delta E=0$, the uncertainty in frequency is $\Delta \omega=\Delta E / \hbar=0$. From the Heisenberg uncertainty relations eq. (4), it follows that the uncertainty in time is $\Delta t=\infty$. This means that one cannot specify the time coordinate hence one can consider the wave function of the electron with the frequency $\omega$ in time. The temporal wave function of the electron has the null speed with respect to the electron $v=0$. Hence, the uncertainty in the space coordinate is $\Delta r=v / \Delta t=0$. This means that the temporal wave function of electron cannot be specified in space. Likewise the spatial wave function of photon the temporal wave function of electron is not suitable to define the speed of light.

There is a problem with interpretation of the time-energy uncertainty relation and the temporal wave function, e.g. (Bush, 2007; Bostrom, 2003) and reference therein. In quantum mechanics time is not an observable but mere a parameter that is different from position being an observable. Therefore, one can introduce the spatial wave function normalized over position space but cannot the temporal wave function. Nevertheless, there are attempts to modify quantum mechanics in a way of introducing the temporal wave function, e.g. (Bush, 2007; Bostrom, 2003) and reference therein. We shall use the temporal wave function putting aside the problem of interpretation.

One can define the temporal wave function of the photon emitted (absorbed) by the electron through the temporal wave function of the electron (virtual photon associated with the electron). Accordingly, the phase of the photon emitted (absorbed) by the electron is defined through the phase of the electron. Take the electron in the stationary state with the energy $E$ that gives the temporal wave function of the electron with the frequency $\omega=E / \hbar$. Let the electron emit (absorb) a photon with the energy $E$ at the time $t_{1}$ and another photon with the energy $E$ at the time $t_{2}$. The phase difference is defined at the time $t_{2}$ between the phase of the second photon and the phase of the electron (virtual photon)

$$
\Delta \phi=\phi_{2}-\phi_{1}=\omega\left(t_{2}-t_{2}\right)=0 .
$$


Although the photons are emitted (absorbed) by the electron at different times $t_{1}$ and $t_{2}$ one determines no phase difference between the photons because they are emitted (absorbed) by the same electron. Note that the temporal wave function of the electron is defined in some point of space $r$ with the uncertainty in the space coordinate $\Delta r=0$. Therefore, comparison of the phases of the photons can occur through the temporal wave function of the same electron in the point of space $r$. This differs from the interaction of the electron and the photon which occurs at the radius $\Delta r=\hbar /(2 k)$ for the time $\Delta t=\hbar /(2 c k)$.

Consider the Michelson-Morley experiment in a frame moving with the velocity $v$ with respect to the CMB frame. Under emission (absorption) of the photons, the electron of the source (receiver) assigns the phases to the photons going along the legs. Although the times of emission (absorption) of the photons are different one determines no phase difference between the photons because the phase difference eq. (6) is determined by means of the temporal wave function of the same electron. Despite the difference of travel time for the photons between two legs one cannot measure this difference as a phase shift that may explain the null result of the Michelson-Morley experiment without invoking the Lorentz transformation.

\section{Conclusion}

We have considered the quantum mechanical model of electromagnetic waves wherein the photon is treated as a particle moving with the speed of light with respect to the CMB frame. The spatial wave function of the photon is introduced as a wave accompanying the photon, with an infinite speed of spreading throughout the whole space at some moment of time. Then, the phase difference between the source and receiver is defined instantaneously and does not depend on the velocity of the frame. The temporal wave function of the photon emitted (absorbed) by the electron is defined through the temporal wave function of the electron (virtual photon associated with the electron) at some point of space. Then, the phases of the different photons defined through the phase of the electron are the same although the moments of time of emission (absorption) of the photons are different. We have studied the Michelson-Morley experiment with use of both the spatial and temporal wave functions of photon and obtained the null result in both the cases. Thus, one can explain the null result of the Michelson-Morley experiment within the quantum mechanics framework without invoking the Lorentz transformation.

According to special relativity (Pauli, 1958) coordinates of space and time follow the Lorentz transformation (LT), $r^{\prime}=r \mathrm{LT}, t^{\prime}=t \mathrm{LT}$, while the wave vector and frequency of electromagnetic wave follow the inverse Lorentz transformation $k^{\prime}=k \mathrm{LT}^{-1}, \omega^{\prime}=\omega \mathrm{LT}^{-1}$. Here the non-primed values are the proper ones while the primed values are the apparent ones. The phase of electromagnetic wave is Lorentz invariant, $\phi^{\prime}=\omega^{\prime} t^{\prime}-k^{\prime} r^{\prime}=\omega t-k r=\phi$, that explains the null result of the Michelson-Morley experiment.

Explanation of the null result of the Michelson-Morley experiment within the quantum mechanics framework allows Galilean invariance of the electromagnetic wave. One may treat the phases given by eq. (5) and eq. (6) as Galilean invariant. That is both observers in a privileged and in a moving frames determine the same space (time) coordinate difference and the same wave vector (frequency). The wave vector (frequency) of the electromagnetic wave emitted in a moving frame is Lorentz shifted in a privileged frame (Doppler effect). In (Khokhlov, 2008) the assumption was made that electromagnetic wave behaves in a different way under propagation and under interaction with the source (receiver). Under propagation electromagnetic wave is Galilean invariant while under interaction with the source (receiver) is Lorentz invariant. Then, we may apply the conventional special relativity under interaction of the electromagnetic wave with the source. After emission the wave vector (frequency) of the electromagnetic wave is the same for both observers in a privileged and in a moving frames. That is Galilean invariance of the phase under propagation of the electromagnetic wave means $\phi=k^{\prime} r=\omega^{\prime} t=\mathrm{Gal}$ inv.

In (Wesley, 1980) Einstein particle dynamics and Maxwell-Lorentz electrodynamics in a moving system was derived without assuming special relativistic kinematics. This means that one can develop the special relativistic dynamics while assuming Galilean invariance. In (Khokhlov, 2009; 2010) the Lorentz transformation for the electromagnetic wave was derived while assuming Galilean invariance.

\section{References}

Bell, J.S. (1989). Speakable and Unspeakable in Quantum Mechanics. Cambridge: Cambridge Univ. Press.

Bohm, D. \& Hiley, B. J. (1993). The undivided universe. London: Routledge.

Bostrom, K. (2003). Quantizing time, arXiv:quant-ph/0301049.

Bush, P. (2007). The time-energy uncertainty relation. In J. G. Muga, R. Sala Mayato \& I. L. Egusquiza (Eds.), Time in quantum mechanics. (2nd ed.). Berlin: Springer-Verlag, (Chapter 3). 
Busch, P. \& Shilladay, C. R. (2006). Complementarity and uncertainty in Mach-Zehnder interferometry and beyond. Phys. Rep., 435, 1-31.

Cahill, R. T. \& Kitto, K. (2003). Michelson-Morley experiments revisited and the cosmic background radiation preffered frame. Apeiron, 10, 104-117.

Cardone, F. \& Mignani, R. (2004). Energy and geometry - an introduction to deformed special relativity. Singapore: World Scientific.

Christov, C. I. (2006). On the material invariant formulation of Maxwell's displacement current. Found. Phys., 36, 1701-1717.

Consoli, M. \& Costanzo, E. (2004). From classical to modern ether-drift experiments: the narrow window for a preffered frame. Phys. Lett. A, 333, 355-363.

Conway, J. \& Kochen, S. (2006). The free will theorem. Found. Phys., 36, 1441-1473.

de Abreu, R. \& Guerra, V. (2008). The principle of relativity and the indeterminacy of special relativity. Eur. $J$. Phys., 29, 33-52.

Dirac, P. A. M. (1958). The Principles of Quantum Mechanics. London: Oxford University Press.

Duffy, M. C. (2004). Geometrized space-time \& the relativistic world ether. In M. C. Duffy (Ed.), Physical Interpretations of Relativity Theory IX, Proceedings Vol. 1 (p.p. 158-202) Liverpool: PD Publications.

Dunning-Davies, J. (2005). A re-examination of Maxwell's electromagnetic equations. Prog. Phys., 3, 48-50.

Gezari, D. Y. (2009). Experimental basis for special relativity in the photon sector, arXiv: 0912.3818.

Hatch, R. R. (2004). Those scandalous clock. GPS Solutions, 8, 67-73.

Holland, B. R. (1994). The quantum theory of motion. Cambridge: Cambridge Univ. Press.

Khokhlov D. L. (2008). Michelson-Morley experiment within the quantum mechanics framework. Concepts of Physics, 5(1), 159-163.

Khokhlov, D. L. (2009). On the non-invariance of the electromagnetic field. Galilean Electrodynamics, 20, SI East 1, 18-19.

Khokhlov, D. L. (2010). Transformation for the energy flux of the electromagnetic wave. Apeiron, 17, 73-80.

Kholmetskii, A. L. (2003). On the non-invariance of the Faraday law of induction. Apeiron, 10, 32-39.

Lineweaver, C. H. et al. (1996). The dipole observed in the COBE DMR 4 year data. Astrophys. J., 470, 38-42.

Landau, L. D. \& Lifshitz, E. M. (1976). The classical theory of fields. (4th ed.). Oxford: Pergamon.

Levy, J. (2003). From Galilei to Lorentz... and beyond. Montreal: Apeiron.

Munera, H. A. (1998). Michelson-Morley experiments revisited: systematic errors, consistency among different experiments, and compatibility with absolute space. Apeiron, 5, 37-53.

Nikolic, H. (2007). Quantum mechanics: Myths and facts. Found. Phys., 37, 1563-1611.

Pauli, W. (1958). Theory of Relativity. New York: Pergamon.

Pospelov, M. \& Romalis, M. (2004). Lorentz invariance on trial. Physics Today, July, 40-46.

Rembielinski, J. \& Smolinski, K. A. (2009). Quantum preferred frame: does it really exist? Eur. Phys. Lett., 88, 10005.

Salart, D. et al. (2008). Testing spooky action at a distance. Nature, 454, 861-864.

Sato, M. (2004). Proposal of Michelson-Morley experiment via single photon interferometer: Interpretation of Michelson-Morley experimental results using de Broglie-Bohm picture, arXiv:physics/0411217.

Selleri, F. (1996). Noninvariant one-way velocity of light. Found. Phys., 26, 641-664.

Shimony, A. (2004). Bell's theorem. In E. N. Zalta (Ed.), Stanford Encyclopedia of Phylosophy, [Online] Available: http://plato.stanford.edu/entries/bell-theorem.

Spavieri, G. (2006). Electromagnetic momentum, magnetic model of light and effects of the Aharonov-Bohm type. Eur. Phys. J. D, 39, 157-166.

Su, C.-C. (2001). A local ether model of propagation of electromagnetic wave. Eur. J. C, 21, 701-715.

Suarez, A. (2003). Entanglement and time, arXiv:quant-ph/0311004. 
Thornhill, C. K. (2004). The foundations of relativity. Hadronic Journal, 27, 499-508.

Wesley, J. P. (1980). Einstein dynamics without special-relativistic kinematics. Found. Phys., 10, 503-511.

Wheeler, J. A. \& Feinmann, R. P. (1949). Classical electrodynamics in terms of direct interparticle action. Rev. Mod. Phys., 21, 425-433.

Whitney, C. K. (2007). Relativistic dynamics in basic chemistry. Found. Phys., 37, 788-812.

Zhang, Y. Z. (1997). Special relativity and its experimental foundation. Singapore: World Scientific. 